\title{
New Immunological Approaches in Hepatocellular Carcinoma: Glypican-3 (GPC-3) Opportunities and Challenges
}

\author{
Bobby Varghese ${ }^{1}$, Navid Darayan ${ }^{1}$, Cristiana Rastellini $^{1}$, Luca Cicalese ${ }^{1}$, \\ Mauro Montalbano ${ }^{1,2,3 *}$ (D) \\ ${ }^{1}$ Department of Surgery, University of Texas Medical Branch, UTMB, Galveston, USA \\ ${ }^{2}$ Department of Neurology, University of Texas Medical Branch, UTMB, Galveston, USA \\ ${ }^{3}$ Mitchell Center for Neurodegenerative Disease, University of Texas Medical Branch, UTMB, Galveston, USA \\ Email: bovarghe@utmb.edu,nadaraya@utmb.edu,crrastel@utmb.edu, lucicale@utmb.edu, ^mamontal@utmb.edu
}

How to cite this paper: Varghese, B., Darayan, N., Rastellini, C., Cicalese, L. and Montalbano, M. (2020) New Immunological Approaches in Hepatocellular Carcinoma: Glypican-3 (GPC-3) Opportunities and Challenges. Journal of Cancer Therapy, 11, 647-659.

https://doi.org/10.4236/jct.2020.1111055

Received: April 18, 2020

Accepted: November 2, 2020

Published: November 5, 2020

Copyright $\odot 2020$ by author(s) and Scientific Research Publishing Inc. This work is licensed under the Creative Commons Attribution International License (CC BY 4.0).

http://creativecommons.org/licenses/by/4.0/

(c) (i) Open Access

\begin{abstract}
Immunotherapy is one of the strategies to boost natural defenses to fight cancer. Immuno-oncology is an artificial stimulation of the human immune system to recognize and kill selectively neoplastic cells at different stage of transformation. Cancer cells have tumor antigens and the antibody of the immune system, binding them, can detect molecules on their extracellular side of cell membrane. Among these proteins, it is rising in interest and used for early detection of hepatocellular carcinoma (HCC) Glypican-3 (GPC-3) protein. It is a heparan sulfate proteoglycan (HSPG), anchored to the cell membrane of transformed hepatocytes. We investigated its function as key regulator of hepatocytes neoplastic transformation. Noteworthy, GPC-3 protein has been implicated in different pathways from cell growth to cell motility and migration. More recently, GPC-3 has been evaluated as a useful marker for HCC due to its increased expression in the liver during tumorigenesis and its absence in normal liver. Immunotherapy that targets GPC-3 domains and its connected proteins are currently under investigation. These new biomarkers may hold potential for the detection and treatment of HCC and other diseases in which GPC-3 may be overexpressed and/or play a crucial role. This review will summarize the current knowledge regarding the active immunotherapy developed to treat HCC and it will evaluate aspects of GPC-3 (structure and biology) as advantages and potential pitfalls for considering it as a valuable immunotherapeutic target. We also elaborated the current literature with the aim to better understand its biological interactions at a molecular and cellular level to identify alternative or combined targets, due to the existing gap in the literature surrounding GPC-3. The role GPC-3 plays in
\end{abstract}


the hepatocellular carcinoma phenotype can be targeted for a novel immunotherapy strategy that can specify cell-mediated destruction of neoplastic cell that spares normal liver tissue, and it can be exploited as a new serum marker to trend for diagnosis and disease progression measurements. We believe further investigation of its functions and structure, including alternative cellular localizations, is necessary to evaluate GPC-3 as valuable target to cure this cancer.

\section{Keywords}

Glypican-3, Hepatocellular Carcinoma, Immunotherapy

\section{Introduction}

Hepatocellular carcinoma is the most common primary liver malignancy. In 2015 there were 854,000 new cases and 810,000 deaths due to hepatocellular carcinoma worldwide. Subsequently, this malignancy has a 12.1 per 100,000 age-adjusted mortality rate, and it is ranked as the sixth most common incident cancer and the fourth leading cause of cancer death [1]. In terms of the global burden of liver cancer, East Asia, Asia Pacific, and Sub-Saharan Africa demonstrated the highest age-adjusted incidence and mortality rates of all regions. The incidence rate of hepatocellular carcinoma has almost doubled in nations such as the United States and Canada with a high sociodemographic index over years of 1990 to 2015 [1].

Usually presenting as a sequela to chronic inflammation and cirrhosis, hepatocellular carcinoma is strongly associated with viral hepatitis, alcohol use, and non-alcoholic fatty liver disease (NAFLD) [2]. Hepatitis B virus (HBV) accounts for $33 \%$ of hepatocellular carcinoma mortality, with alcohol and hepatitis $\mathrm{C}$ virus (HCV) accounting for $30 \%$ and $21 \%$ respectively [1]. NAFLD has been identified as an independent risk factor for hepatocellular carcinoma in North America, Europe, and well developed regions of East Asia, but the lack of clinical data has made it difficult to discern where in the course of the disease hepatocellular carcinoma becomes a complication [3].

\section{Current Therapy}

The standard regimen for the treatment of hepatocellular carcinoma follows the Barcelona Clinic Liver Cancer Staging (BLCS) model that dictates treatment based on tumor progression, size, vascular invasion, metastases, and degree of concurrent liver disease. Early stage lesions are best treated with resection given that liver function is intact. In the setting of chronic liver disease, portal hypertension, or lesions that complicate the local anatomy, a liver transplant can be considered if the patient meets the Milan Criteria (single lesion $<5 \mathrm{~cm}$ or up to 3 lesion $<3 \mathrm{~cm}$ without vascular involvement or metastases to other organs). 
However, this strategy is not always efficacious due to the social constraints and the limited reserve of viable organs available for patients [2] [4]. Radiofrequency ablation is indicated in instances where tumors are localized to the livery only and a liver transplant or resection is not indicated due to concurrent liver disease

[5]. Cases with intermediate staging are indicated for the use of radiofrequency embolization (TARE) and trans arterial chemoembolization (TACE) [2] [4] [6] [7]. Both methods yield similar survival outcomes, but TARE provides greater utility than TACE for patients because it can be performed in an outpatient facility, requires less rounds of treatment, can be used on patients with portal vein thrombosis, and generally has fewer occurrences of nausea, vomiting, abdominal pain, and fever [6]. Advanced stage hepatocellular carcinoma, as defined by BLCS as being multinodular or extra-hepatic with portal invasion but with preserved liver function, is currently managed with the use of systemic treatment. This includes the use of oral multi-tyrosine kinase inhibitors like sorafenib and regorafenib. Sorafenib inhibits angiogenesis by targeting PGFR and VEGFR pathways as well as proliferation and growth pathways such as ERK/MEK and BRAF and Jak-STAT [8]. The use of sorafenib in metastatic hepatocellular carcinoma has demonstrated modest increases in overall survival and time to progression with significant risk of adverse side effects such as dermatologic and gastrointestinal complications [2] [5] [6].

\section{Immunotherapy in HCC}

The immunological disarray that characterizes hepatocellular carcinoma makes immunotherapy a promising frontier for future treatment possibilities. Under normal physiological conditions, $\mathrm{T}$ cell proliferation is actively induced while $\mathrm{T}$ cell activation is suppressed to allow for the steady clearance of pathogens without eliciting a strong immune response. The effective eradication of neoplastic cells requires the appropriate costimulation signals from antigen presenting cells for $\mathrm{T}$ cells to target neoplastic antigens. In the setting of chronic inflammation, as seen in chronic alcoholic hepatitis and viral hepatitis, proliferation of T-cells continues without the required costimulation signals which lead to an ineffective immune response. The blunted cell-mediated immune response is sustained by T-cells expressing high levels inhibitory receptors such as PD-1 and CTLA-4 that inhibit costimulation. Cytokine imbalances characterized by increased levels of inhibitory cytokines such as IL-10 and TGF $\beta$ and the suppression of IFN $\gamma$, TNF, and IL-1 also lead to immunosuppression. [9]

Current investigations into immunotherapy have revealed immune checkpoint regulators, PD1/PD-L1 and CTLA-4/CD86, as potential therapeutic targets for hepatocellular carcinoma. PD-1L is a thought to be induced by IFN $\gamma$ as a means of protecting normal cells by binding PD-1 on T-cells which leads to inhibiting the immunological response. Neoplastic cells constitutively express PD-L1 to take advantage of this mechanism to evade the immune response. [10] The CTLA-4 pathway is predominately utilized by $\mathrm{T}$ reg cells as a means to at- 
tenuate the T-cell response by expressing CTLA-4 that competes with B7 for the CD86 receptor on T-cells. CTLA-4 binds up CD86 and inhibits the cancer-primed $\mathrm{T}$ cells from targeting neoplastic cells. [9]

Nivolumab is a human IgG4 monoclonal antibody to PD-1 that is currently approved for the treatment of melanoma. Phase III randomize clinical trials are currently being done comparing nivolumab to sorafenib for hepatocellular carcinoma (NCT 02576509). Pembrolizumab is another IgG4 monoclonal antibody against PD-1 that has been approved for hand neck squamous cell carcinoma, non-small cell lung cancer, refractory classic Hodgkins Lymphoma and metastatic melanoma. Phase III trials are currently underway to determine its efficacy when combined with standardized best supportive care in prolonging the progression free survival rate of advanced hepatocellular carcinoma (NCT 02702401). Tremelimumab and Durvalumab combination therapy for hepatocellular carcinoma is currently in phase III clinical trials to examine the efficacy of PD-1 and CTLA-4 blockade (NCT 02519348). Clinical Trials details are summarized in Table 1.

\section{Glypican-3 Role in Tumorigenesis}

Glypican-3 (GPC-3) is a $70 \mathrm{kDa}$ transmembrane heparan sulfate proteoglycan family (HSPG), which has been shown to regulate growth activity and release of growth factors. During embryogenesis it is ubiquitously expressed in all tissues to serve as a negative growth regulator of growth through interactions with the canonical and non-canonical Wnt pathway, Shh pathway, and FGF pathways via heperan sulfate chains on GPC-3. In adult tissue it is normally expressed only in kidney, lung, ovaries, and mammary glands while suppressed in other tissues [11].

In hepatocellular carcinoma however, GPC-3 demonstrates a paradoxical function as a marker of increased neoplastic growth and invasion. We recently demonstrated that inhibiting its expression, HCC cells reduce their proliferation and invasiveness capacity. GPC-3 is also involved in the oncogenic transformation of hepatocytes with a marked increment of its expression. We characterized primary hepatocytes from different location of human cirrhotic liver with HCC and we observed differential expression of GPC-3 coupled with different growth rates of cells [12] [13] [14]. The regulation of GPC-3 has been linked to the

Table 1. Current clinical trials evaluating the efficacy of immunotherapy for cancer treatment.

\begin{tabular}{cccccc}
\hline Name & Isotype & Target & NCT Number & Study Design & $\begin{array}{c}\text { Clinical Trial } \\
\text { Phase }\end{array}$ \\
\hline Nivolumab & IgG4 & PD-1 & 02576509 & RCT & III \\
Pembrolizumab & IgG4 & PD1 & 02702401 & RCT & III \\
Tremelimumab & IgG2 & CTLA-4 & 02519348 & RCT & II \\
Durvalumab & IgG1 & PD-L1 & & & \\
\hline
\end{tabular}


upregulation of the oncogene c-myc. Upregulation of c-myc is a common feature of many human tumors, and it has been demonstrated by $\mathrm{Li}$ and coworkers that c-myc can upregulate GPC-3 expression by binding to the GPC-3 promoter, and in turn c-myc activity is dependent on GPC-3 in a positive-feedback manner [15] (Figure 1). GPC-3 has also been shown to affect cell morphology via interactions with the Hippo/YAP pathway (Figure 1). Normal hepatic tissue expresses the tetraspanin CD81 which interacts with GPC-3 to downregulate the Hippo/YAP pathway. HCV induces factors that can mimic the effects of CD81, and subsequently bind GPC-3 and inhibit cell proliferation. However, in the setting of inflammation, cells that have lost CD81 expression escape infection by $\mathrm{HCV}$, are predisposed to becoming neoplastic due to unregulated Hippo/YAP activity [16]. De novo loss of CD81 is feature of HCC secondary to chronic liver disease, and in this context will lead to disinhibition of the Hippo/YAP pathway, which in turn upregulates GPC-3 (Figure 1). GPC-3 has been shown to sequestering Wnt proteins to the plasma membrane and subsequently facilitating the interaction between Wnt and Frizzled which activates the Wnt pathway [17] (Figure 2). Inhibiting with specific antibodies for O-linked heparan sulfate (HS)

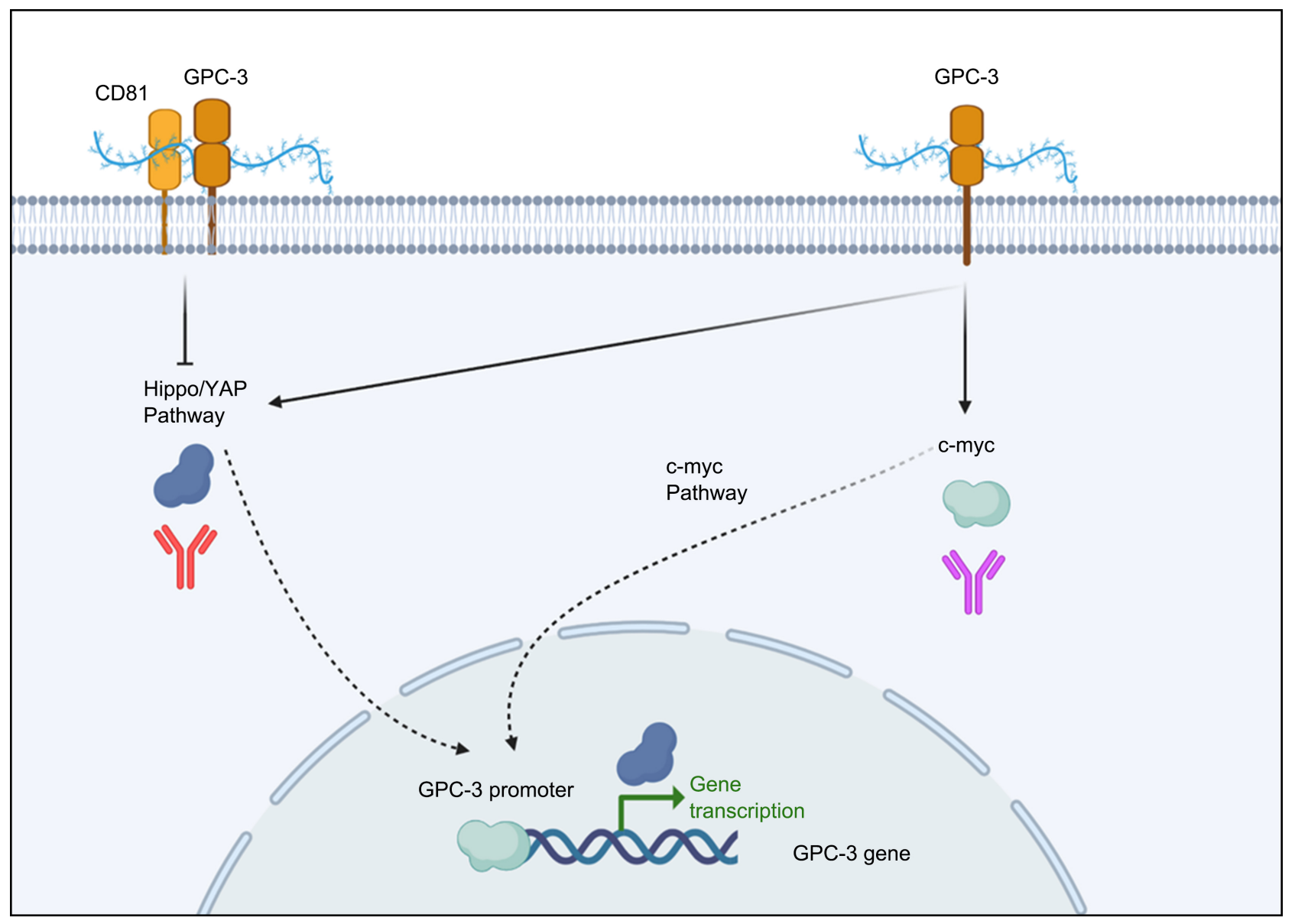

Figure 1. Activation of c-Myc and Hippo/YAP Pathways modulate GPC-3 expression. Dysregulation of CD81 along with aberrant activation of Wnt, Hippo/Yap, and c-myc contribute to the overexpression of GPC-3 gene. Both YAP and c-myc represent possible new immune-targets for HCC. 


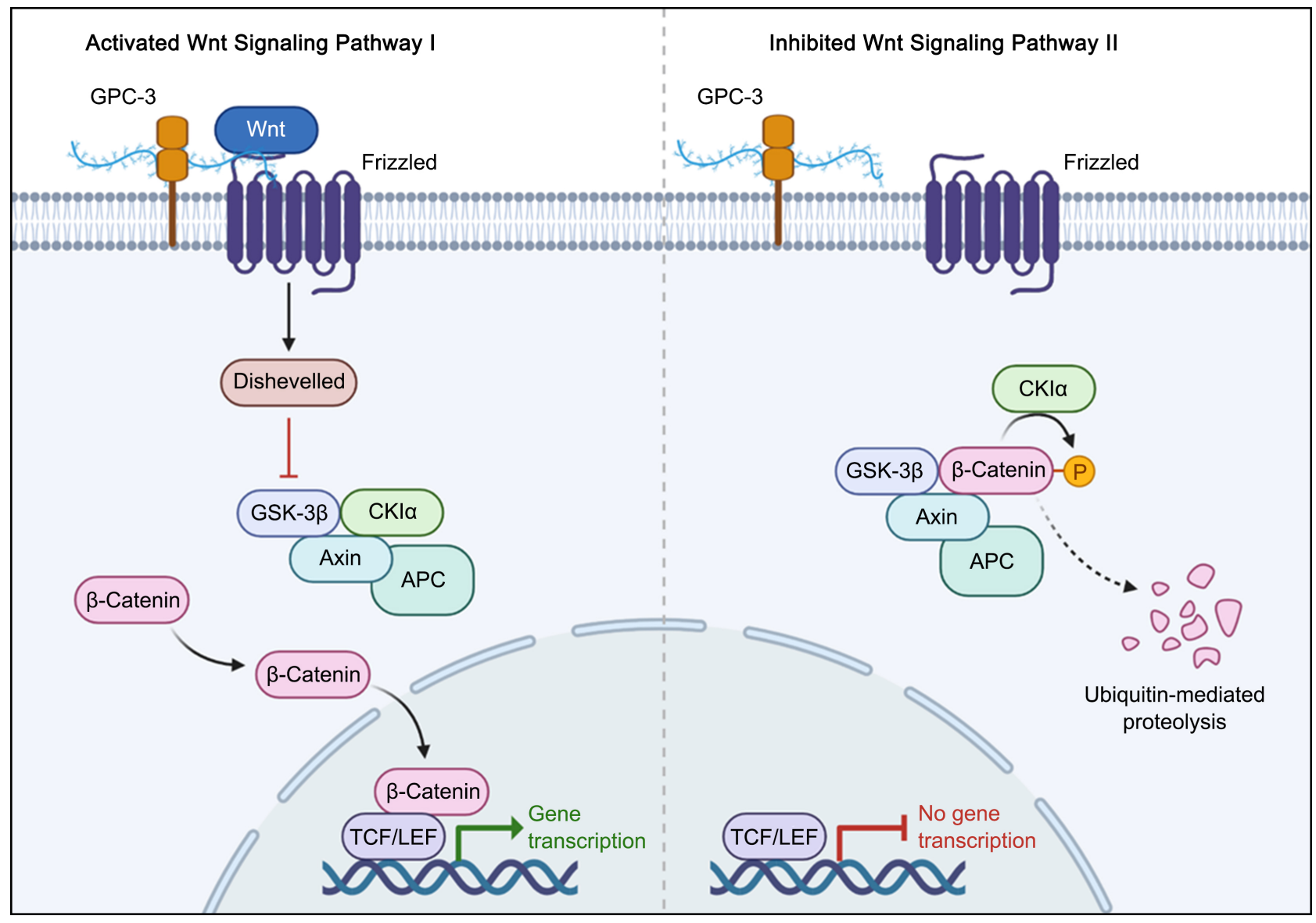

Figure 2. GPC-3 and overstimulation of Wnt-Frizzled Pathway. (I) GPC-3 recruits Wnt to the plasma membrane and binds Frizzled receptor, which ultimately leads to dysregulation of the $\beta$-Catenin degradation, which translocates in the nucleus and active transcription of genes involved in proliferation, evasion of apoptosis, migration and invasion. (II) In the absence or not interaction of GPC-3 with Frizzled receptor, Wnt factor is not sequestered to the membrane, which allows for normal inhibition of $\beta$-Catenin.

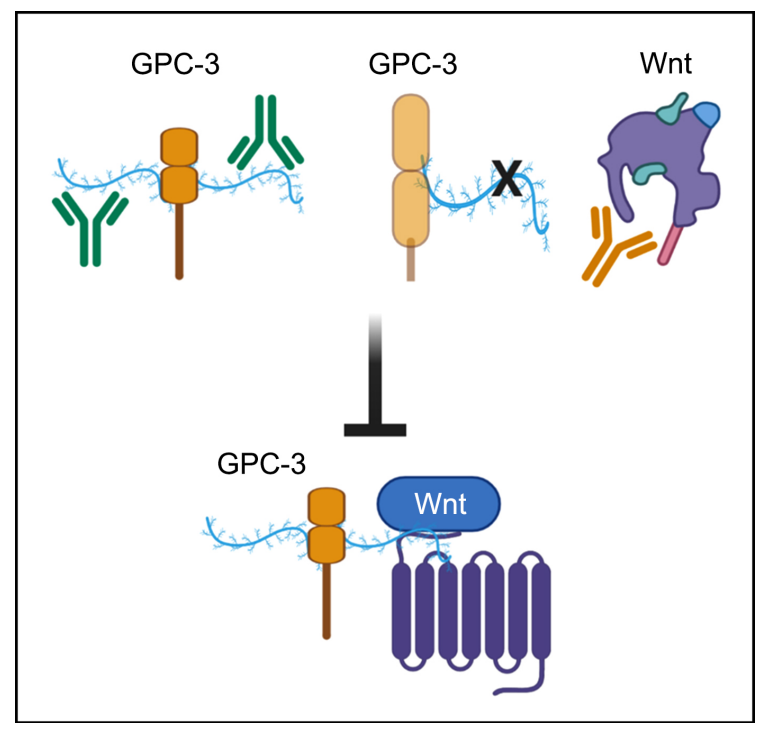

Figure 3. Prevent interaction of GPC-3 with Wnt. Antibody specific for site of interactions between GPC-3 and Wnt could prevent interaction between them. The inhibition of this interaction inactivate the Wnt/Frizzled cascade in HCC cells. 
side chains could prevent the interaction of GPC-3 with Wnt deploying the activation of Frizzled mediate signal (Figure 3).

\section{Utility of GPC-3 in Treatment of HCC}

The expression profile of GPC-3 in HCC provides a potentially unique avenue for treatment or early detection of neoplastic lesions. Wang and coworkers demonstrated that overexpression of IncRNA FENDRR interacted with the promoter of GPC-3 and lead to the downregulation of GPC-3 and subsequently inhibited neoplastic proliferation and migration [18]. Bi and coworkers has demonstrated that the use of bispecific $\mathrm{T}$ cell engagers against $\mathrm{CD} 3$ and GPC-3 has demonstrated potently cytotoxic activity against GPC-3 positive tumors in NOD/SCID mice [19]. Currently clinical trials are exploring the use of chimeric antigen receptors (CAR) against GPC-3 in advanced HCC, though the results are still pending on the efficacy of these treatments (NCT02395250, NCT02723942).

The gold standard of diagnosing HCC is currently limited to imaging techniques using ultrasound or CT alongside elevated serum alpha fetoprotein (AFP), which has spurred the search for novel biomarkers for detecting neoplastic changes unique to HCC. Cross-sectional analysis conducted by Tahon and coworkers demonstrates that GPC-3 at a detection limit of $1.5 \mathrm{ng} / \mathrm{ml}$ is superior to AFP at $5.9 \mathrm{ng} / \mathrm{ml}$ in detecting smaller grade $\mathrm{HCC}$, and in some cases $\mathrm{HCC}$ patients with a negative AFP were positive for GPC-3. However, GPC-3 alone fails to have absolute sensitive when compared to triphasic CT. When GPC-3 and AFP were used in combination, the sensitivity for diagnosis increased [20]. A case-control study by Chen and coworkers compared the diagnostic efficacy of GPC-3, AFP, Arginase 1 (ARG1), and hepatocyte paraffin antigen 1 (HepPar-1) in confirming extra-hepatic metastasis of HCC in cases where HCC has yet to be diagnosed. The study surmised that GPC-3 was more sensitive at $89.2 \%$ compared to AFP at $82.6 \%$, but GPC-3 yielded a lower sensitivity at $94.8 \%$ with AFP at $100 \%(\mathrm{P}<0.001)$ [21].

Though the properties of GPC-3 and its role in the development HCC have to be clearly defined, its potential as a target for cancer therapy and diagnostics has been repeatedly demonstrated in published literature. The diagnostic properties of GPC-3 have shown promising results in increasing the current specificity of detecting HCC and in detecting lesions that are at an earlier stage. Current immunological therapies have explored preventing immunologic evasion by tumor cells as a more efficient therapeutic approach, and GPC-3 can serve as inimitable target for specifying cytotoxic activity against HCC lesions (Figure 4) not only within the liver but also in distant metastases.

\section{Conclusions}

Unfortunately, the high incidence of HCC has not been encountered with efficacious treatment options, and as a result prognosis continues to be poor. Current therapeutic options like surgery, chemotherapy and radiotherapy have only 


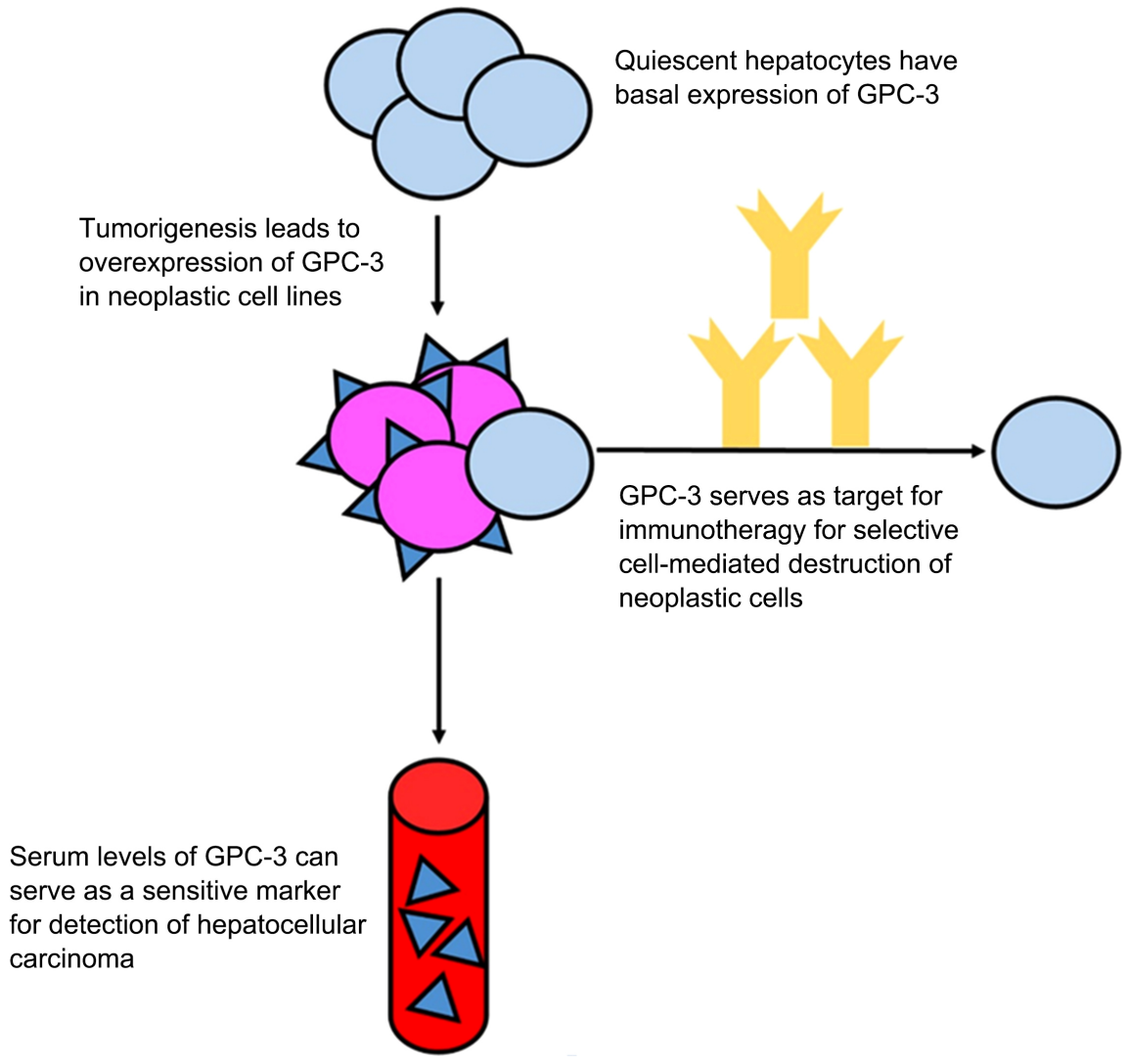

Figure 4. Utilization of GPC-3 for Immunotherapy and Diagnosis of HCC. GPC-3 is upregulated in neoplastic hepatocytes which can targeted for immunotherapy and as a novel serum marker for diagnosis of HCC.

modest and uncertain success and they do not prevent recurrences in the patients. New treatment modalities to prolong survival and to minimize the risk of adverse response are urgently needed for patients with advanced HCC due to the increment of patients with HCC in the United States. In this context, tumor immunotherapy is a promising and novel treatment strategy that may lead to improvements in both treatment-associated toxicity and overall mortality. The strategies have developed in part through genomic studies that have yielded candidate target molecules (mainly proteins) and in part through basic biology studies that have defined the pathways and cell types regulating immune response. In the last years GPC-3 is an emerging target in diverse investigational immunotherapies, with attention on cancer vaccines, human or humanized antibodies, bispecific antibodies, immunotoxins and chimeric antigen receptor (CAR) T cells. Vaccines based of GPC-3 peptide-based prevent recurrence of HCC after surgery. Several monoclonal antibodies including GC33 [22], YP7 [23], HN3-mPE24 [24] and HS20 [25], each with a distinct epitope for GPC-3, are being tested in preclinical or clinical stages. GC33 and YP7 recognize the C-terminal domain of GPC-3. HN3 targets a cryptic Wnt binding site in the core protein of GPC-3. HS20 binds a Wnt binding domain on the heparan sulfate chain. The HN3 and HS20 antibodies inhibit Wnt/Yap signaling suppressing 
HCC cell growth. To improve anti-tumor efficacy, anti-GPC3 immunotoxins and CAR-T cell therapies [26] have been developed. The GC33 CAR T cells inhibit established HCC xenograft tumors in mice [22] [27]. Several CARs targeting different epitopes of GPC3 (including HN3) are being tested and new strategy as GPC-3-specific antibody drug conjugates have been proposed [28]. Ongoing preclinical and clinical studies will help define the utility of GPC3 as a therapeutic target for liver cancer therapy soon.

There is a critical requirement to discover and develop new therapeutic approaches that can be targeted against molecular targets on HCC cells and protecting normal hepatocytes from neoplastic transformation. Immunotherapy has become a big and popular topic of interest in exploring new treatment options for advanced cancer, however current therapies take a generalized approach by focusing on reversing immune escape mechanisms deployed by cancer cells. The mechanistic disadvantage to these therapies therefore involves developing nondiscriminatory autoimmune diseases. GPC-3 provides a potential and new molecular target for cancer therapy in patients that have been diagnosed with HCC since it is overexpressed in most HCC tumors. As described previously we observed that GPC-3 is cleaved by Furin enzyme generating different cleaved domains, it is important to investigate the roles and cellular localization of this domain and eventually target specifically them because they can be involved in different functions during the tumorigenesis. It has been shown that GPC-3 plays a role in progression of HCC through activation of the canonical Wnt pathway and other pathways, which provides a novel perspective against diagnosing and treating HCC. With the current search for immunological markers that can direct the cytotoxic efforts of the immune system towards cancer cells, GPC-3 presents itself as a novel target for using immunotherapy for HCC. Furthermore, soluble GPC-3 has been shown to be a valuable tumor marker for diagnosing and tracking progression of HCC. With its diagnostic advantages as well as its ability for tumorigenesis, GPC-3 has the potential to be a focus of a new frontier in the management and treatment of HCC.

\section{Supplemental Information}

All table and figures materials were presented in this manuscript are original. In particular the pathways represented in Figure 1, Figure 2 and Figure 3 were generated using BioRender Software. Figure 4 has been generated using PathVisio Software. All the authors read and proofread the manuscript and no conflict of interest is present.

\section{Conflicts of Interest}

The authors declare no conflicts of interest regarding the publication of this paper.

\section{References}

[1] Global Burden of Disease Liver Cancer Collaboration (2017) The Burden of Prima- 
ry Liver Cancer and Underlying Etiologies from 1990 to 2015 at the Global, Regional, and National Level: Results from the Global Burden of Disease Study 2015. JAMA Oncology, 3, 1683-1691.

[2] Forner, A., Reig, M. and Bruix, J. (2018) Hepatocellular Carcinoma. The Lancet, 391, 1301-1314. https://doi.org/10.1016/S0140-6736(18)30010-2

[3] Degasperi, E. and Colombo, M. (2016) Distinctive Features of Hepatocellular Carcinoma in Non-Alcoholic Fatty Liver Disease. The Lancet Gastroenterology \& Hepatology, 1, 156-164. https://doi.org/10.1016/S2468-1253(16)30018-8

[4] Hartke, J., Johnson, M. and Ghabril, M. (2017) The Diagnosis and Treatment of Hepatocellular Carcinoma. Seminars in Diagnostic Pathology, 34, 153-159. https://doi.org/10.1053/j.semdp.2016.12.011

[5] Eggert, T. and Greten, T.F. (2017) Current Standard and Future Perspectives in Non-Surgical Therapy for Hepatocellular Carcinoma. Digestion, 96, 1-4. https://doi.org/10.1159/000464282

[6] Mokdad, A.A., Hester, C.A., Singal, A.G. and Yopp, A.C. (2017) Management of Hepatocellular in the United States. Chinese Clinical Oncology, 6, 21. https://doi.org/10.21037/cco.2017.04.04

[7] Cheraghvandi, L., Silva, M., Cheng, C., et al. (2016) Single-Step Combined Laparoscopic Management of Hepatocellular Carcinoma with Simultaneous Radio Frequency Ablation and Trans-Arterial Embolization in Unresactable Lesions. Journal of Cancer Therapy, 7, 979-985. https://doi.org/10.4236/jct.2016.713095

[8] Cheng, A.-L., Kang, Y.-K., Chen, Z., et al. (2009) Efficacy and Safety of Sorafenib in Patients in the Asia-Pacific Region with Advanced Hepatocellular Carcinoma: A Phase III Randomised, Double-Blind, Placebo-Controlled Trial. The Lancet Oncology, 10, 25-34. https://doi.org/10.1016/S1470-2045(08)70285-7

[9] Harding, J.J., Dika, I. and Abou-Alfa, G.K. (2016) Immunotherapy in Hepatocellular Carcinoma: Primed to Make a Difference? Cancer, 122, 367-377. https://doi.org/10.1002/cncr.29769

[10] Patel, S.P. and Kurzrock, R. (2015) PD-L1 Expression as a Predictive Biomarker in Cancer Immunotherapy. Molecular Cancer Therapeutics, 14, 847-856. https://doi.org/10.1158/1535-7163.MCT-14-0983

[11] Montalbano, M., Georgiadis, J., Masterson, A.L., et al. (2017) Biology and Function of Glypican-3 as a Candidate for Early Cancerous Transformation of Hepatocytes in Hepatocellular Carcinoma (Review). Oncology Reports, 37, 1291-1300. https://doi.org/10.3892/or.2017.5387

[12] Montalbano, M., Rastellini, C., McGuire, J.T., et al. (2018) Role of Glypican-3 in the Growth, Migration and Invasion of Primary Hepatocytes Isolated from Patients with Hepatocellular Carcinoma. Cellular Oncology, 41, 169-184.

https://doi.org/10.1007/s13402-017-0364-2

[13] Montalbano, M., Curcuru, G., Shirafkan, A., Vento, R., Rastellini, C. and Cicalese, L. (2016) Modeling of Hepatocytes Proliferation Isolated from Proximal and Distal Zones from Human Hepatocellular Carcinoma Lesion. PLoS ONE, 11, e0153613. https://doi.org/10.1371/journal.pone.0153613

[14] Montalbano, M., Rastellini, C., Wang, X., Gorgun, T., Eltorky, M.A., Vento, R. and Cicalese, L. (2016) Transformation of Primary Human Hepatocytes in Hepatocellular Carcinoma. International Journal of Oncology, 48, 1205-1217. https://doi.org/10.3892/ijo.2015.3312

[15] Li, L., Jin, R., Zhang, X., et al. (2012) Oncogenic Activation of Glypican-3 by c-Myc 
in Human Hepatocellular Carcinoma. Hepatology, 56, 1380-1390. https://doi.org/10.1002/hep.25891

[16] Xue, Y., Mars, W.M., Bowen, W., Singhi, A.D., Stoops, J. and Michalopoulos, G.K. (2018) Hepatitis C Virus Mimics Effects of Glypican-3 on CD81 and Promotes Development of Hepatocellular Carcinomas via Activation of Hippo Pathway in Hepatocytes. The American Journal of Pathology, 188, 1469-1477. https://doi.org/10.1016/j.ajpath.2018.02.013

[17] Akutsu, N., Yamamoto, H., Sasaki, S., et al. (2010) Association of Glypican-3 Expression with Growth Signaling Molecules in Hepatocellular Carcinoma. World Journal of Gastroenterology, 16, 8. https://doi.org/10.3748/wjg.v16.i28.3521

[18] Wang, B., Xian, J., Zang, J., et al. (2019) Long Non-Coding RNA FENDRR Inhibits Proliferation and Invasion of Hepatocellular Carcinoma by Down-Regulating Glypican-3 Expression. Biochemical and Biophysical Research Communications, 509, 143-147. https://doi.org/10.1016/j.bbrc.2018.12.091

[19] Bi, Y., Jiang, H., Wang, P., et al. (2017) Treatment of Hepatocellular Carcinoma with a GPC3-Targeted Bispecific T Cell Engager. Oncotarget, 8, 52866-52876. https://doi.org/10.18632/oncotarget.17905

[20] Tahon, A.M., El-Ghanam, M.Z., Zaky, S., et al. (2018) Significance of Glypican-3 in Early Detection of Hepatocellular Carcinoma in Cirrhotic Patients. Journal of Gastrointestinal Cancer, 50, 434-441. https://doi.org/10.1007/s12029-018-0095-2

[21] Chen, D., Li, Z., Song, Q., Qian, L., Xie, B. and Zhu, J. (2018) Clinicopathological Features and Differential Diagnosis of Hepatocellular Carcinoma in Extrahepatic Metastases. Medicine, 97, e13356. https://doi.org/10.1097/MD.0000000000013356

[22] Zhu, A.X., Gold, P.J., El-Khoueiry, A.B., et al. (2013) First-in-Man Phase I Study of GC33, a Novel Recombinant Humanized Antibody against Glypican-3, in Patients with Advanced Hepatocellular Carcinoma. Clinical Cancer Research, 19, 920-928. https://doi.org/10.1158/1078-0432.CCR-12-2616

[23] Phung, Y., Gao, W., Man, Y.G., Nagata, S. and Ho, M. (2012) High-Affinity Monoclonal Antibodies to Cell Surface Tumor Antigen Glypican-3 Generated through a Combination of Peptide Immunization and Flow Cytometry Screening. MAbs, 4, 592-599. https://doi.org/10.4161/mabs.20933

[24] Wang, C., Gao, W., Feng, M., Pastan, I. and Ho, M. (2017) Construction of an Immunotoxin, HN3-mPE24, Targeting Glypican-3 for Liver Cancer Therapy. Oncotarget, 8, 32450-32460. https://doi.org/10.18632/oncotarget.10592

[25] Gao, W., Kim, H., Feng, M., et al. (2014) Inactivation of Wnt Signaling by a Human Antibody That Recognizes the Heparan Sulfate Chains of Glypican-3 for Liver Cancer Therapy. Hepatology, 60, 576-587. https://doi.org/10.1002/hep.26996

[26] Liu, X., Wen, J., Yi, H., et al. (2020) Split Chimeric Antigen Receptor-Modified T Cells Targeting Glypican-3 Suppress Hepatocellular Carcinoma Growth with Reduced Cytokine Release. Therapeutic Advances in Medical Oncology, 12. https://doi.org/10.1177/1758835920910347

[27] Fleming, B.D., Urban, D.J., Hall, M.D., et al. (2019) Engineered Anti-GPC3 Immunotoxin, HN3-ABD-T20, Produces Regression in Mouse Liver Cancer Xenografts through Prolonged Serum Retention. Hepatology, 71, 1696-1711. https://doi.org/10.1002/hep.30949

[28] Fu, Y., Urban, D.J., Nani, R.R., et al. (2019) Glypican-3-Specific Antibody Drug Conjugates Targeting Hepatocellular Carcinoma. Hepatology, 70, 563-576.

https://doi.org/10.1002/hep.30326 


\section{Abbreviations}

Hepatocellular Carcinoma, HCC; Barcelona Clinic Liver Cancer Staging: BCLCS; Glypican-3: GPC-3; Hepatitis B virus: HBV; Hepatitis C Virus: HCV; non-alcoholic fatty liver disease: NAFLD; radiofrequency embolization, TARE; Transarterial chemoembolization, TACE; Number Clinical trial, NCT; heparan sulfate proteoglycan, HSPG; chimeric antigen receptors, CAR; Randomized clinical trial, RCT. 


\section{Appendix}

\section{Search Terms}

glypican 3 AND hepatocellular carcinoma

glypican 3 expression and hepatocellular carcinoma

(hepatocellular carcinoma or liver cancer) immunotherapy

(hepatocellular carcinoma or liver cancer) and current treatment

(hepatocellular carcinoma OR liver cancer) and current treatment

(hepatocellular carcinoma or liver cancer) and current treatment

(hepatocellular carcinoma or liver cancer) and current therapy

(hepatocellular carcinoma or liver cancer) and diagnosis

hepatocellular carcinoma treatment

(hepatocellular carcinoma OR primary liver cancer) and mortality hepatocellular carcinoma OR primary liver cancer

hepatocellular carcinoma epidemiology

(hepatocellular carcinoma OR liver cancer) AND epidemiology

Search liver cancer epidemiology

\section{Selection Criteria}

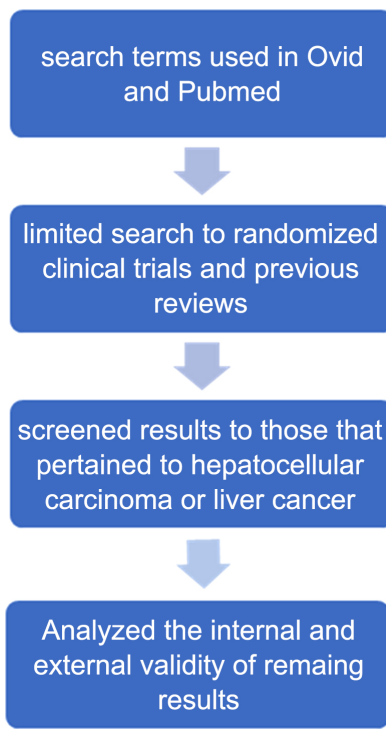

